\title{
Effects of Microwave and Water Incorporation on Natural Deep Eutectic Solvents (NADES) and Their Extraction Properties
}

\author{
Analía V. Gómez ${ }^{1,2,3}$, Atanu Biswas ${ }^{3 *}$, Carmen C. Tadini ${ }^{1,2}$, Karl Vermillion ${ }^{3}$, Megan Buttrum ${ }^{3}$ and Huai \\ N. Cheng ${ }^{4 *}$ \\ ${ }^{1}$ University of São Paulo, Escola Politécnica, Department of Chemical Engineering, \\ Main Campus, São Paulo, 05508-010, SP, Brazil \\ ${ }^{2}$ University of São Paulo, FoRC/NAPAN - Food Research Center, São Paulo, SP, Brazil \\ ${ }^{3}$ USDA Agricultural Research Service, National Center for Agricultural Utilization Research, \\ Peoria, IL, United States \\ ${ }^{4}$ USDA Agricultural Research Service, Southern Regional Research Center, New Orleans, LA, United States \\ Email: atanu.biswas@ars.usda.gov; hn.cheng@ars .usda.gov
}

\begin{abstract}
One of the common processes employed in food technology and preparation is the extraction of specific components from agro-based materials. Although many methodologies are known, continued improvements are still desirable. A recent new development is the use of natural deep eutectic solvents (NADES) for extraction. In this work, we studied NADES/water combinations and found them to be viable alternatives to conventional solvents for extraction of polar ingredients in foods. The NADES/water solvents are eco-friendly, inexpensive, chemically inert, and biodegradable. Moreover, we found microwave heating to facilitate the preparation of NADES/water solvents. Selected NADES/water systems were characterized with respect to viscosity, refractive indices, electrical conductivity, water activity, and NMR. In addition, we utilized the microwave process to facilitate the extraction of sugars in a food product with a NADES/water system. The microwave process was found to be efficient, save time, and decrease energy usage. Thus, the combination of NADES/water/microwave is a versatile method that can provide improved extractions in food systems.
\end{abstract}

Keywords: Extraction, NADES, deep eutectic solvent, microwave, NMR, conductivity, viscosity.

\section{Introduction}

Green chemistry is the design of chemical products and processes that reduce or eliminate the generation of hazardous substances [1-3]. This concept has become popular in the current business and regulatory climate with the increasing emphasis on safer chemistry, product stewardship, and sustainability of natural resources. Whereas green chemistry is helpful in chemical product and processes, it takes on even greater significance in food applications, where food safety, human health, chemical toxicity, and environmental pollution are critical.

One of the common processes used in food processing is the extraction of specific substances from agro-based raw materials $[4,5]$. For example, triglyceride oil from soybean, cottonseed, corn, and rice bran can be extracted with hydrocarbon solvents [6-9]. Likewise the essential oils from biological plants and flowers can be extracted for use as flavour and fragrance [10-12]. Phytochemicals can be extracted from fruits, berries, beans, and other agro-based materials, using (for example) alcohol or alcohol/water mixtures [13-15]. In view of the current interest in green chemistry, new or improved extraction methodologies that do not involve organic solvents are desirable. A promising recent solvent approach involves the natural deep eutectic solvents (NADES) [16-19].

A NADES solvent contains two or three natural, renewable, and biodegradable natural components, which form a eutectic mixture because of self-association and/or specific interactions among the solvent components [16-19]. A distinctive feature of the eutectic mixture is the melting point, which is lower than the melting points of the individual components; the NADES of interest are liquids from room temperature to $100{ }^{\circ} \mathrm{C}$. Examples of NADES include mixtures of choline chloride/1,2-propanediol, 
choline chloride/glycerol, citric acid/glucose, malic acid/glucose, and malic acid/beta-alanine. A disadvantage of NADES is their high viscosity compared with conventional organic or aqueous solvents. For the purpose of food extraction, the high viscosity of NADES would certainly make the process very difficult.

It has been reported that the addition of water can decrease the viscosity of NADES [20, 21]. The aim of this study is to explore the addition of water to NADES as solvents for the extraction of polar ingredients from agro-based materials. In addition, we have studied the use of microwave, both for the preparation of NADES solvents and for extraction, in order to improve the efficiency of NADES-based extraction processes.

\section{$2 \quad$ Materials and Methods}

\section{$2.1 \quad$ Materials}

All compounds used as NADES components in this work were acquired from Sigma Aldrich (St. Louis, MO, USA). Deuterated dimethylsulfoxide $\left(\mathrm{d}_{6}\right.$-DMSO) came from Cambridge Isotope Laboratories, Inc., Andover, MA. Green bananas were acquired from a local grocer.

\subsection{Sample Preparation and Evaluation}

Several NADES systems were prepared by heating with constant stirring (at $400 \mathrm{rpm}$ ) until a clear liquid was formed; water was added until a reasonably workable viscosity was obtained (Table 1). Both conventional and microwave heating was used. In addition, the effects of water dilution (0-90\%) on viscosity, refractive index, density, water activity and electrical conductivity of NADES were evaluated. These NADES solvents were stored in sealed bottles at room temperature away from sunlight. They remained to be liquid under this condition for at least 4 weeks.

Table 1. Composition and abbreviations for NADES studied in this work.

\begin{tabular}{ccccc}
\hline Component 1 & Component 2 & Component 3 & Component molar ratio & Designation \\
& & & & \\
\hline Malic acid & Glucose & Water & $1: 1: 3$ & $\mathrm{MA} / \mathrm{Glu}$ \\
Malic acid & Fructose & Water & $1: 1: 3$ & $\mathrm{MA} / \mathrm{Fru}$ \\
Malic acid & b-Alanine & Water & $1: 1: 3$ & $\mathrm{MA} / \mathrm{BA}$ \\
Citric acid & Glucose & Water & $1: 1: 2$ & $\mathrm{CA} / \mathrm{Glu}$ \\
Citric acid & Fructose & Water & $1: 1: 2$ & $\mathrm{CA} / \mathrm{Fru}$ \\
Urea & Glucose & Water & $1: 1: 2$ & $\mathrm{U} / \mathrm{Glu}$ \\
Urea & Fructose & Water & $1: 1: 2$ & $\mathrm{U} / \mathrm{Fru}$ \\
Glucose & Choline Cl & Water & $2: 3: 3$ & $\mathrm{Glu} / \mathrm{CC}$ \\
Fructose & Choline Cl & Water & $2: 3: 3$ & Fru/CC \\
\hline
\end{tabular}

The measurement of electrical conductivity was carried out at $25{ }^{\circ} \mathrm{C}\left( \pm 2{ }^{\circ} \mathrm{C}\right)$ with a $\mathrm{S} 470$ SevenExcellence $^{\mathrm{TM}}$ conductivity meter equipped with an InLab731 ISM sensor (Mettler Toledo, Columbus, OH, USA). The refractive index was employed as a measure of the soluble solids content; a NADES solvent system was diluted with variable amounts of water, and the refractive index was determined at $20{ }^{\circ} \mathrm{C}$ using an Abbe 3L refractometer (Bausch \& Lomb, Rochester, NY, USA). Dynamic viscosity of a NADES solution was measured at $25^{\circ} \mathrm{C}\left( \pm 2^{\circ} \mathrm{C}\right)$ using an ARES LS-1 control-strain rheometer (TA Instruments, New Castle, DE, USA). The determination of the density of NADES was conducted at $40{ }^{\circ} \mathrm{C}$ with vibrating tube density meter (Model DMA ${ }^{\mathrm{TM}} 4500 \mathrm{M}$, Anton Paar, Graz, Austria). This device entailed a measurement cell with a U-shaped borosilicate glass tube and two integrated Pt 100 platinum thermometers and Peltier elements that controlled and registered the temperature. The determination of the water activities of NADES solvents was carried out at $25^{\circ} \mathrm{C}( \pm$ $2{ }^{\circ} \mathrm{C}$ ) with an Aqua-Lab water activity meter (Series 3TE, Decagon Devices Inc., Pullman, WA, USA). 


\subsection{Extraction of Soluble Sugars with NADES Solvents}

Soluble sugars from banana puree were extracted with a microwave system (Ethos 1600 Advance Microwave Labstation, Milestone, Sorisole, Italy) using a 100-mL extractor vessel. Banana puree (3 g) was weighed into the reactor vessel, and soluble sugars were extracted with $30 \mathrm{~g}$ of a NADES system (molar ratio of 1:1:3 MA:BA:water), and two solvents (ethanol and water) via microwave-assisted extraction. After extraction, samples were centrifuged at $4{ }^{\circ} \mathrm{C}$ for $20 \mathrm{~min}$ in a Heraeus Multifuge X1R centrifuge (Thermo Fisher Scientific, Langenselbold, Germany) at 10,000x g to separate the supernatant from the residue. An aliquot of each supernatant obtained was analyzed for total sugar by the phenolsulfuric acid method [22].

\subsection{NMR Characterization}

NMR spectra were acquired on a Bruker DRX 400 spectrometer (Karlsruhe, Germany). Standard operating conditions were used with $30^{\circ}$ pulse angle and $3 \mathrm{~s}$ between pulses. The sample of choline chloride/1,2-propanediol/water was run without a deuterated lock solvent. The sample containing choline chloride/glycerol had $\mathrm{d}_{6}$-DMSO added as the lock solvent. All chemical shifts were referenced to tetramethylsilane at $0 \mathrm{ppm}$. Spectral assignments were made using chemical shifts of related structures, additive shift rules [23], and automated shift prediction software [24].

\section{$3 \quad$ Results and Discussion}

\subsection{Incorporation of Water in NADES}

As we started to work on NADES systems, we quickly noticed a problem. The NADES tended to be rather viscous and difficult to handle. In agreement with the literature [20, 21], we found the incorporation of water on NADES solvents to have a significant positive effect. In fact, viscosity showed an exponential decrease with an increase in water content (Figure 1). The following trend was observed for five NADES systems:

$\mathrm{CA} / \mathrm{Glu}>>>\mathrm{MA} / \mathrm{Glu} \sim \mathrm{Glu} / \mathrm{CC}>\mathrm{U} / \mathrm{Glu}>>\mathrm{MA} / \mathrm{BA}$

Although Figure 1 gives only the effects observed for five NADES systems, the same trends observed for glucose-NADES samples were also found for fructose-NADES samples. In addition, we found microwave heating to be the preferred method of preparing the NADES mixtures. On the basis of past work [21], several temperatures and heating times were tested on a few NADES systems. The optimum conditions to achieve a clean liquid NADES that were stable for several weeks were found and shown in Table 2. In all cases the use of microwave heating produced a decrease in the preparation time relative to conventional heating.

Table 2. Comparison between conventional and microwave heating in NADES preparation.

\begin{tabular}{ccccc}
\hline Designation & Molar ratio & Temp $\left({ }^{\circ} \mathrm{C}\right)$ & Conv. Heat Time $(\min )$ & Microwave Time $(\min )$ \\
\hline MA/Glu & $1: 1: 3$ & 70 & 60 & 5 \\
MA/Fru & $1: 1: 3$ & 60 & 60 & 10 \\
MA/BA & $1: 1: 3$ & 60 & 60 & 5 \\
CA/Glu & $1: 1: 2$ & 80 & 120 & 30 \\
CA/Fru & $1: 1: 2$ & 75 & 120 & 30 \\
U/Glu & $1: 1: 2$ & 70 & 60 & 10 \\
U/Fru & $1: 1: 2$ & 60 & 90 & 20 \\
Glu/CC & $2: 3: 3$ & 70 & 120 & 30 \\
Fru/CC & $2: 3: 3$ & 70 & 60 & 15 \\
\hline
\end{tabular}

We then proceeded to measure some of the properties of the NADES/water systems. The electrical conductivity of NADES without the addition of water decreased in the following sequence: $\mathrm{MA} / \mathrm{BA}>>$ Fru/CC $>$ Glu/CC $>>\mathrm{MA} / \mathrm{Fru}>\mathrm{MA} / \mathrm{Glu}>>\mathrm{U} / \mathrm{Fru} \sim \mathrm{U} / \mathrm{Glu} \sim \mathrm{CA} / \mathrm{Glu} \sim \mathrm{CA} / \mathrm{Fru}$. Conductivity of NADES increased as a result of the decreased viscosity, due to the dilution caused by 
the addition of water (Figure 2). Some NADES (Glu/CC, Fru/CC, U/Glu, U/Fru, MA/BA) reached their maximum value of electrical conductivity with $60 \%$ of added water, and the others (MA/Glu, MA/Fru, CA/Glu, CA/Fru) with $80 \%$ of added water (Figure 2). In general, higher solvent conductivity is beneficial for the extraction of polar compounds. Furthermore, the water activity also increased with water addition (Figure 3). Although Figures 2-3 provides the effects observed for five NADES systems, the same trends were also observed for fructose-NADES samples. Since higher water content enhances the conductivity and water activity, higher water content should furnish better efficiencies for the extraction of polar components, and lower water content for less polar components.

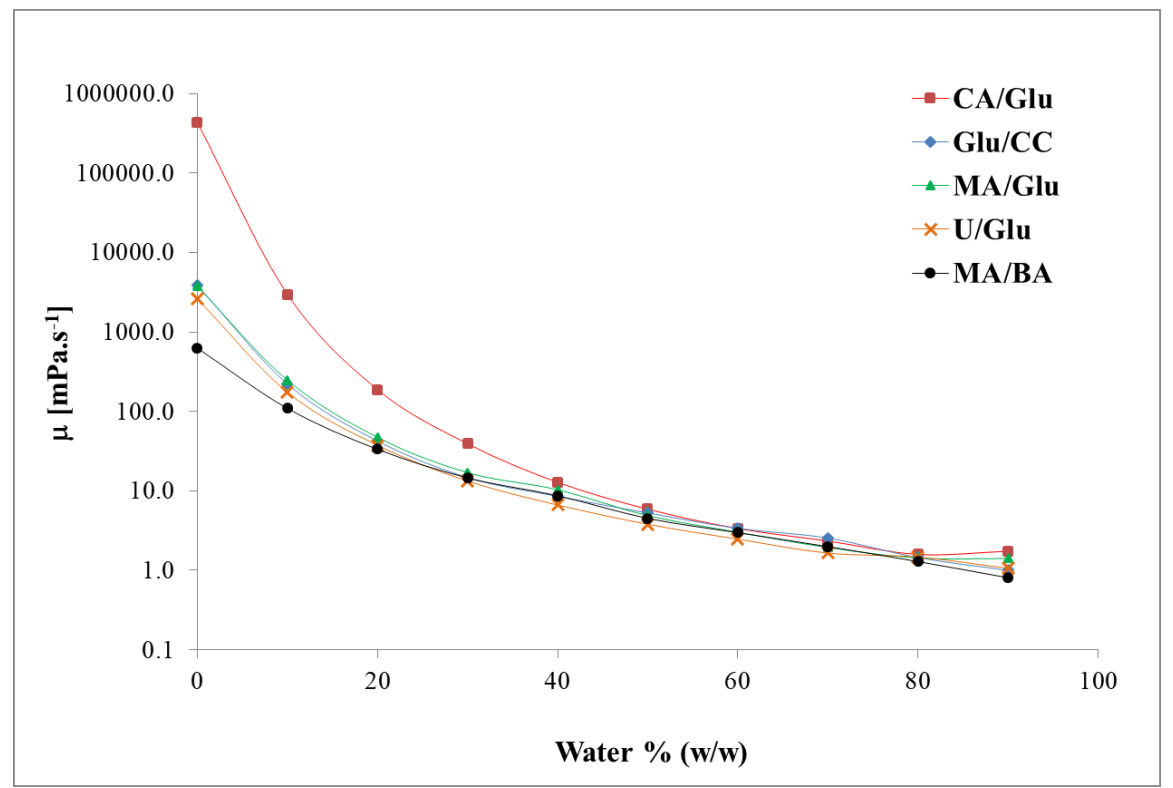

Figure 1. Viscosity $(\boldsymbol{\mu})$ of five NADES systems as a function of added water. The same trends for glucose-NADES samples were also found for fructose-NADES samples.

Density and refractive index decreased linearly as the water content increased, following the function, $\mathrm{y}=-\mathrm{ax}+\mathrm{b}$ (y: density or refractive index, $\mathrm{x}$ : water percentage) (Figure 2, Table 3). Without the addition of water, the highest densities were found for NADES prepared with citric acid/sugar or malic acid/sugar (densities for CA/Glu=1.442 $\mathrm{g} \cdot \mathrm{cm}^{-3}, \mathrm{CA} / \mathrm{Fru}=1.428 \mathrm{~g} \cdot \mathrm{cm}^{-3}$, MA/Glu=1.406 $\mathrm{g} \cdot \mathrm{cm}^{-3}$, $\left.\mathrm{MA} / \mathrm{Fru}=1.396 \mathrm{~g} \cdot \mathrm{cm}^{-3}\right)$. The lowest values were obtained in samples containing choline chloride $\left(\mathrm{Glu} / \mathrm{CC}=1.246 \mathrm{~g} \cdot \mathrm{cm}^{-3}, \mathrm{Fru} / \mathrm{CC}=1.249 \mathrm{~g} \cdot \mathrm{cm}^{-3}\right)$. Sample of MA/BA and those containing urea showed intermediate values $\left(\mathrm{MA} / \mathrm{BA}=1.340 \mathrm{~g} \cdot \mathrm{cm}^{-3}, \mathrm{U} / \mathrm{Fru}=1.381 \mathrm{~g} \cdot \mathrm{cm}^{-3}, \mathrm{U} / \mathrm{Glu}=1.378 \mathrm{~g} \cdot \mathrm{cm}^{-3}\right)$. The lowest refractive index values were found for NADES prepared with malic acid/ $\beta$-alanine (MA/BA), and highest values were obtained for samples with sugar/choline chloride (Glu/CC and Fru/CC) when no dilutions were made to the NADES (Table 3). The fact that the refractive index is higher for samples with less quantity of water can be explained by the presence of molecules that are more polarizable than water. Therefore, the larger the number of polarizable molecules in the sample, the larger was the value of the refractive index.

From the data in Figure 2, NADES/water systems prepared with choline chloride presented the highest conductivities, while NADES with urea showed the lowest values. NADES with choline chloride/sugar and urea/sugar had the highest conductivity at $60 \%$ water dilution. Organic acid/sugar NADES reached the highest value with $80 \%$ water dilution. Consequently, electrical conductivity of NADES, as well as viscosity and density, could be tailored by changing the water content. 

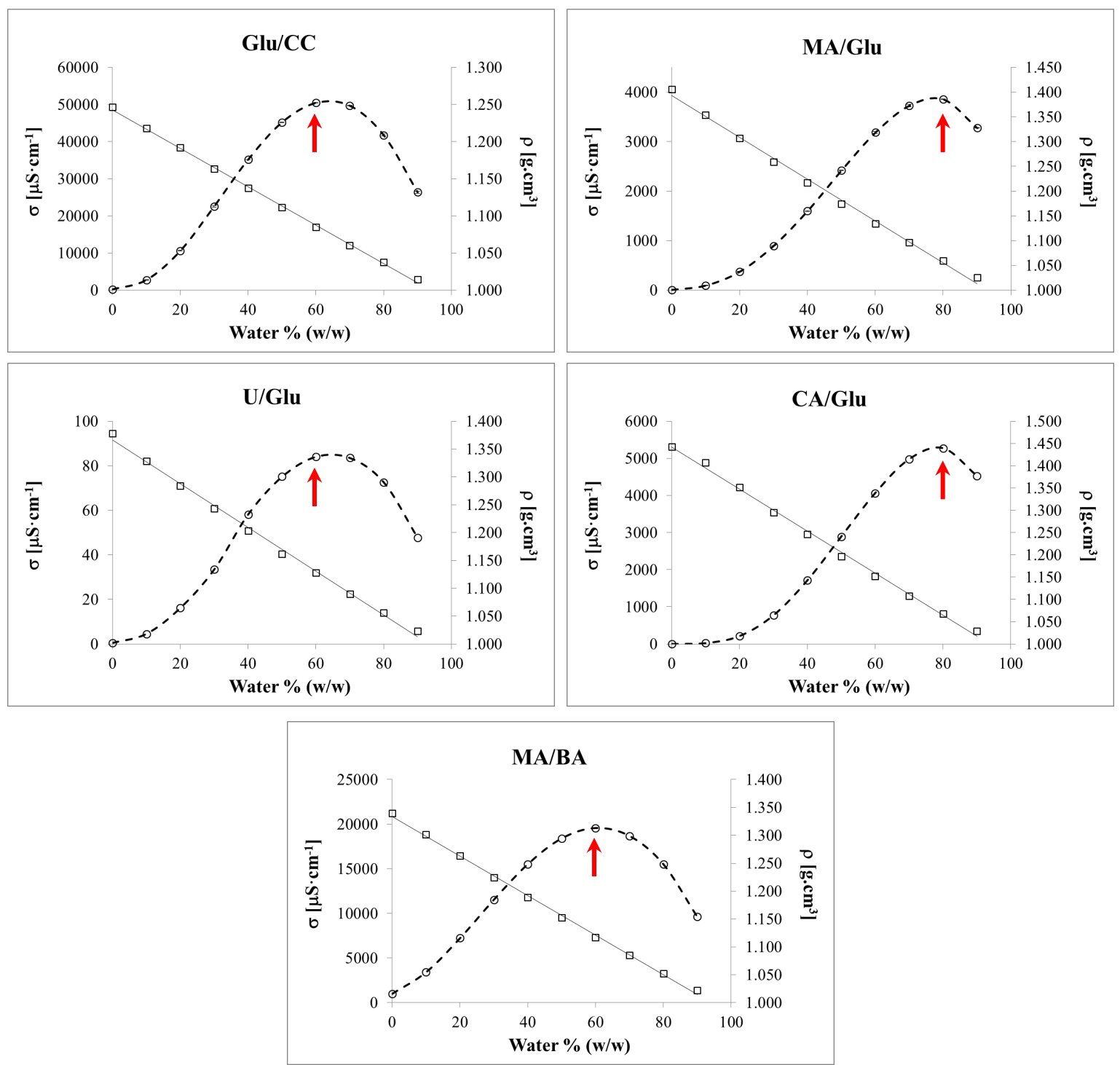

Figure 2. Conductivity ( $\sigma$, dashed curves) and density ( $\rho$, solid lines) of five NADES systems as a function of added water. Red arrows indicate the highest conductivity values. The same trends for glucose-NADES samples were also found for fructose-NADES samples.

Table 3. Refractive index at $20{ }^{\circ} \mathrm{C}$ of NADES diluted with different amounts of water

\begin{tabular}{cccccccccc}
\hline Water content* & MA/Glu & MA/Fru & MA/BA & CA/Glu & CA/Fru & U/ Glu & U/Fru & Glu/CC & Fru/CC \\
\hline $\mathbf{0}$ & 1.483 & 1.480 & 1.473 & 1.499 & 1.494 & 1.498 & 1.497 & 1.503 & 1.502 \\
$\mathbf{1 0}$ & 1.464 & 1.462 & 1.458 & 1.477 & 1.474 & 1.477 & 1.477 & 1.483 & 1.482 \\
$\mathbf{2 0}$ & 1.446 & 1.445 & 1.442 & 1.458 & 1.455 & 1.458 & 1.457 & 1.464 & 1.463 \\
$\mathbf{3 0}$ & 1.429 & 1.428 & 1.426 & 1.440 & 1.437 & 1.439 & 1.439 & 1.445 & 1.444 \\
$\mathbf{4 0}$ & 1.413 & 1.413 & 1.411 & 1.422 & 1.419 & 1.422 & 1.421 & 1.427 & 1.427 \\
$\mathbf{5 0}$ & 1.398 & 1.397 & 1.397 & 1.405 & 1.402 & 1.405 & 1.405 & 1.409 & 1.409 \\
$\mathbf{6 0}$ & 1.384 & 1.383 & 1.383 & 1.389 & 1.387 & 1.389 & 1.388 & 1.392 & 1.392 \\
$\mathbf{7 0}$ & 1.370 & 1.370 & 1.370 & 1.374 & 1.373 & 1.374 & 1.374 & 1.377 & 1.377 \\
$\mathbf{8 0}$ & 1.357 & 1.357 & 1.357 & 1.359 & 1.359 & 1.360 & 1.359 & 1.362 & 1.362 \\
$\mathbf{9 0}$ & 1.345 & 1.345 & 1.345 & 1.346 & 1.345 & 1.346 & 1.346 & 1.347 & 1.347 \\
\hline
\end{tabular}

$*$ Water content $=$ quantity of water added to $\operatorname{NADES}(\%, \mathrm{w} / \mathrm{w})$ 


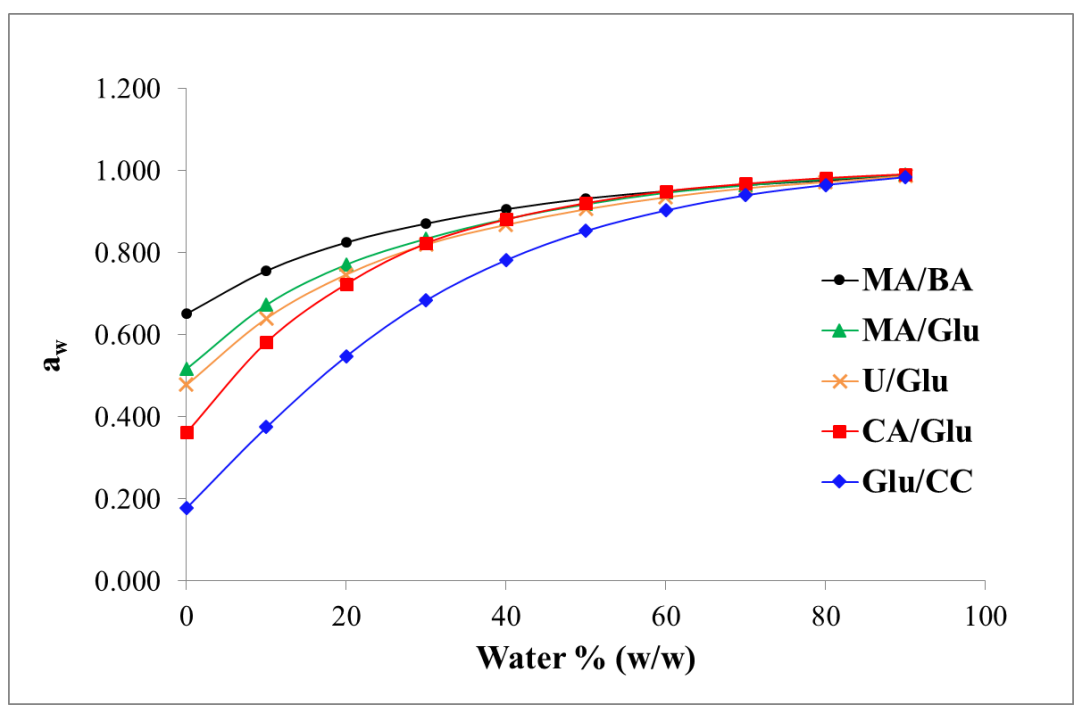

Figure 3. Water activity $\left(a_{w}\right)$ of five NADES systems as a function of added water. The same trends for glucoseNADES samples were found for fructose-NADES samples.

\subsection{NMR Characterization}

In order to understand the interactions of water with the other components of NADES, we studied two selected NADES/water systems with NMR.

\subsubsection{Choline chloride/1,2-propanediol/water (1:1:1) mixture}

Choline chloride and 1,2-propanediol are known to form a NADES system, and the addition of water can decrease the viscosity [21]. The ${ }^{1} \mathrm{H}$ NMR spectrum of this mixture is shown in Figure 4. To help assign the spectrum, we used the two-dimensional (2D) NMR techniques, particularly ${ }^{1} \mathrm{H}$ correlation spectroscopy (COSY) (Figure 5a) and heteronuclear single quantum coherence spectroscopy (HSQC) (Figure 5b). Through 2D HSQC, all the ${ }^{1} \mathrm{H}$ and the ${ }^{13} \mathrm{C}$ peaks were correlated and assigned (Table 4). To confirm our assignments, we did the 2D COSY experiment (Figure 5a). From the 2D plot, it is clear that our assigned peaks for P-2 (3.6 ppm) and P-3 (1.05 ppm) are correlated, thereby verifying our assignments. Previously, Dai et al [21] studied this solvent system and provided NMR assignments. Our assignments agreed with those of Dai et al [21] with one exception. The paper in Dai et al [21] (Table S1) reversed the assignments of $\mathrm{P}-1$ and $\mathrm{P}-2$.

Table 4. NMR chemical shifts and correlations for 1:1:1 (molar) choline chloride/1,2-propanediol/water mixture

\begin{tabular}{|c|c|c|c|c|}
\hline Code & ${ }^{1} \mathrm{H}$ shift (ppm) & ${ }^{13} \mathrm{C}$ shift $(\mathrm{ppm})$ & $\begin{array}{c}\text { ROESY } \\
\text { correlations } \\
\end{array}$ & HOESY correlations \\
\hline $\begin{array}{l}\mathrm{P}-\mathrm{OH} 1 \\
\mathrm{P}-\mathrm{OH} 2\end{array}$ & 4.85 & & $\mathrm{C}-\mathrm{OH}, \mathrm{H}_{2} \mathrm{O}$ & \\
\hline $\mathrm{P}-1$ & 3.35 & 67 & & \\
\hline $\mathrm{P}-2$ & 3.60 & 67.5 & & \\
\hline $\mathrm{P}-3$ & 1.05 & 20 & & \\
\hline $\mathrm{C}-\mathrm{OH}$ & 5.40 & & $\mathrm{P}-\mathrm{OH}, \mathrm{H}_{2} \mathrm{O}, \mathrm{C}-1, \mathrm{C}-3$ & \\
\hline $\mathrm{C}-1$ & 3.95 & 55 & $\mathrm{C}-\mathrm{OH}$ & $\mathrm{C}-2$ \\
\hline $\mathrm{C}-2$ & 3.55 & 67.2 & & $\mathrm{C}-1$ \\
\hline $\mathrm{C}-3$ & 3.25 & 53.8 & $\mathrm{C}-\mathrm{OH}$ & \\
\hline $\mathrm{H}_{2} \mathrm{O}$ & 4.40 & & $\mathrm{C}-\mathrm{OH}, \mathrm{P}-\mathrm{OH} 1, \mathrm{P}-\mathrm{OH} 2$ & \\
\hline $\begin{array}{l}\text { ucture: } \\
\text { des: }\end{array}$ & $\begin{array}{l}\mathrm{HO}-\mathrm{CH}_{2}-\mathrm{CH} \\
\text { P-OH1 P-1 P-2 }\end{array}$ & $\begin{array}{l}\mathrm{OH})-\mathrm{CH}_{3} \\
\text {-OH2 P-3 }\end{array}$ & \begin{tabular}{llll}
\multicolumn{3}{c}{$\mathrm{HO}-\mathrm{CH}_{2}-\mathrm{CH}_{2}-\mathrm{N}^{+}(\mathrm{C}$} \\
$\mathrm{C}-\mathrm{OH}$ & $\mathrm{C}-1$ & $\mathrm{C}-2$
\end{tabular} & \\
\hline
\end{tabular}




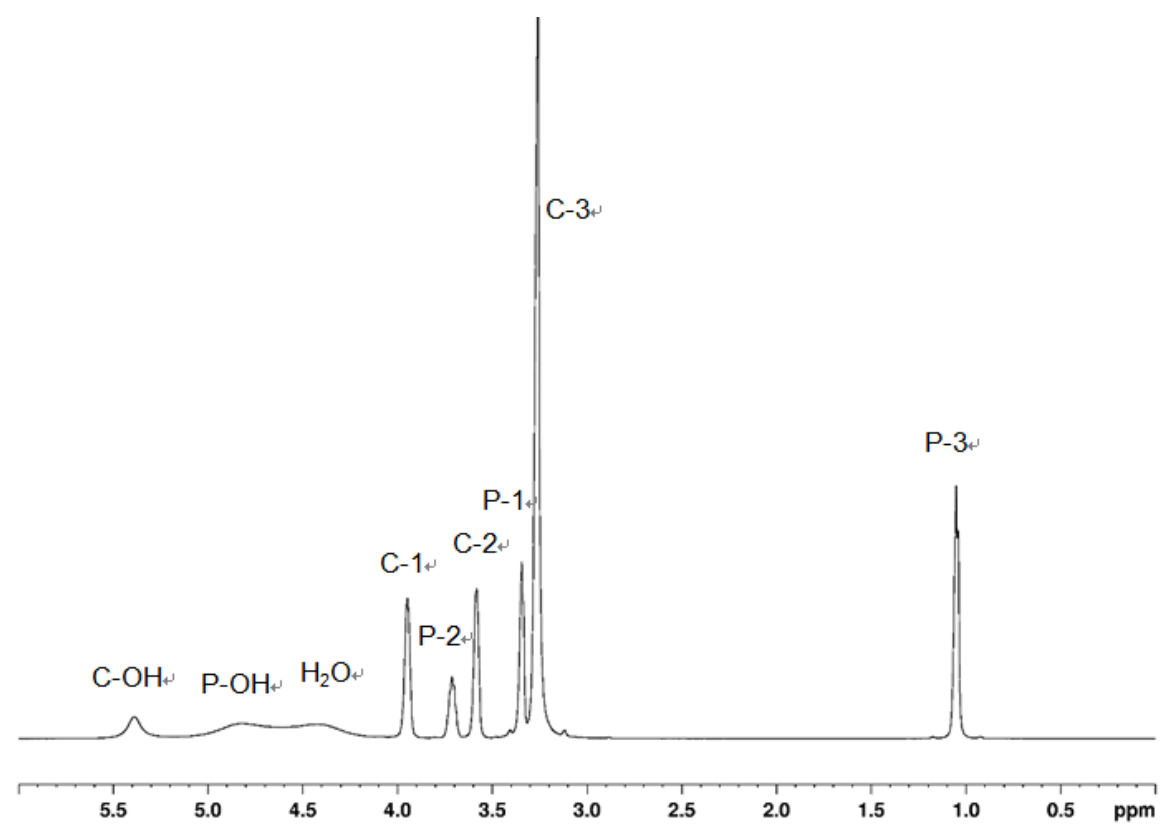

Figure 4. ${ }^{1} \mathrm{H}$ NMR spectrum of choline chloride/1,2-propanediol/water mixture.
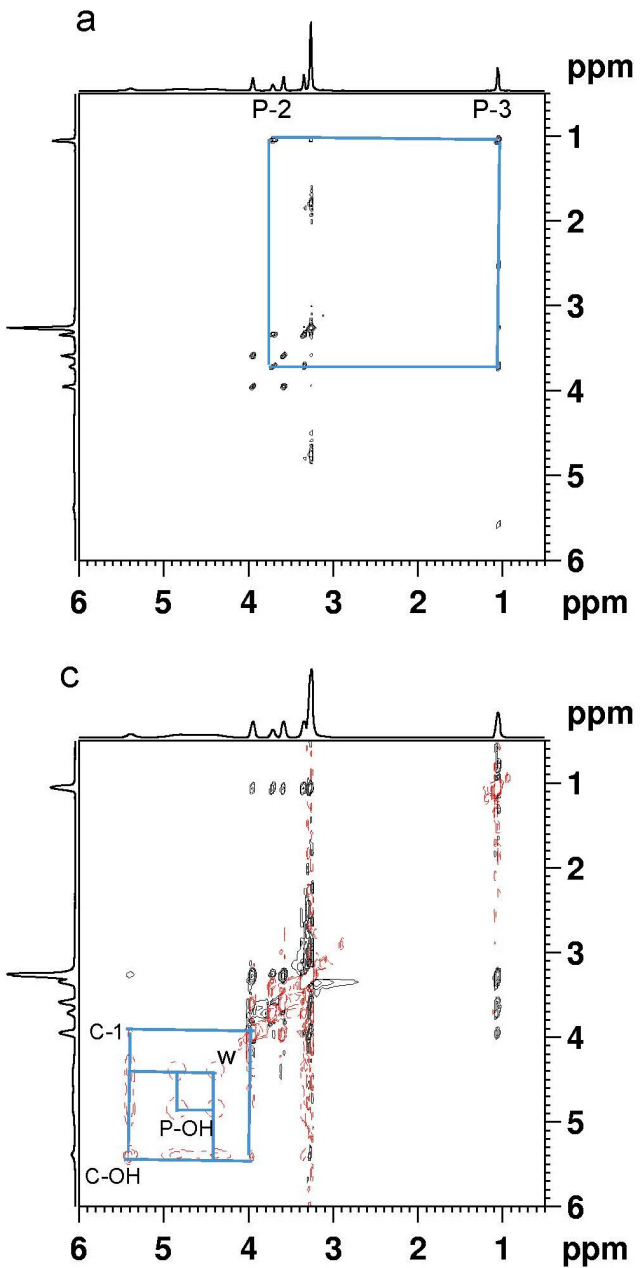
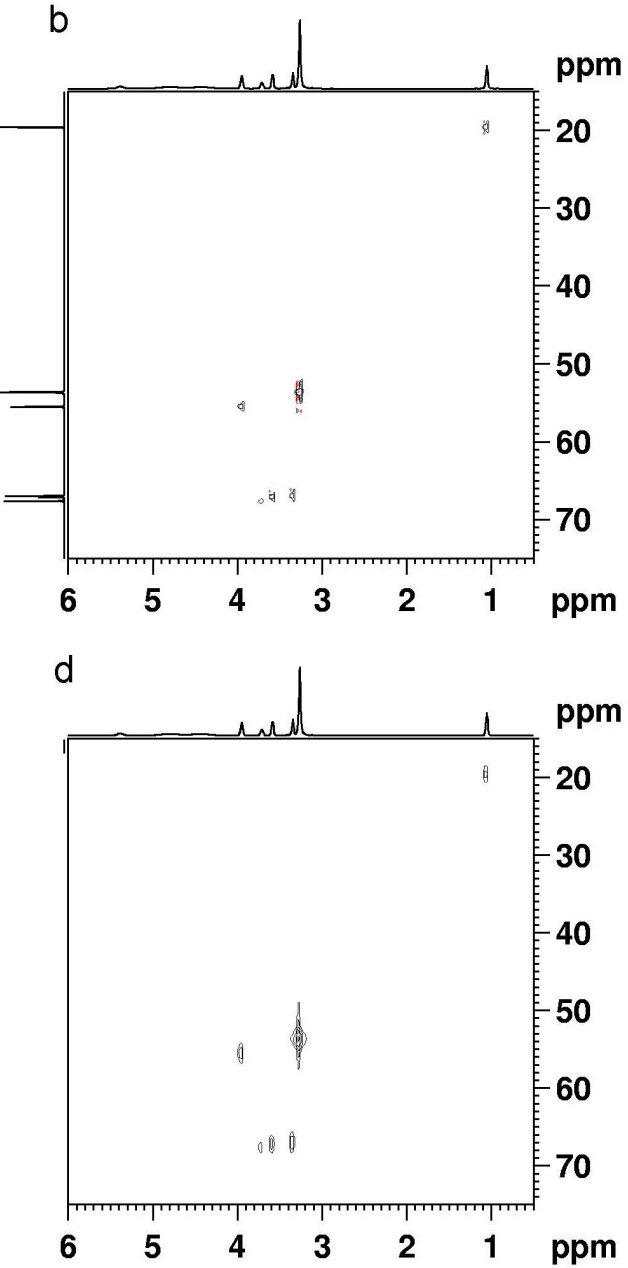

Figure 5. 2D NMR spectra of choline chloride/1,2-propanediol/water mixture: a) COSY, b) HSQC, c) ROESY, d) HOESY. The letter w refers to the water peak. 

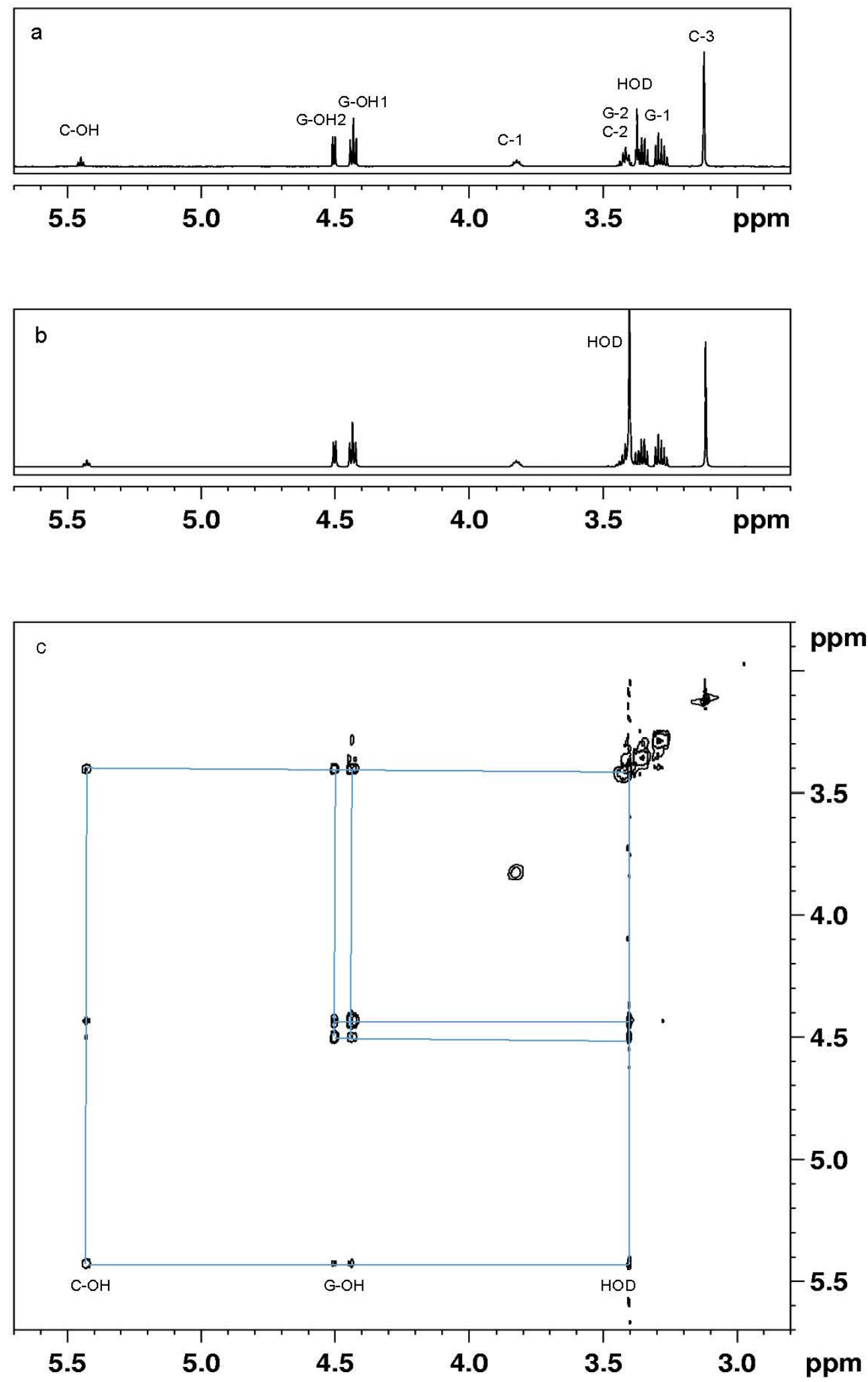

Figure 6. ${ }^{1} \mathrm{H}$ NMR spectra: a) choline chloride/glycerol mixture, b) choline chloride/glycerol with $25 \%$ water, c) NOESY plot of choline chloride/glycerol with $25 \%$ water
structure: $\quad \mathrm{HO}-\mathrm{CH}_{2}-\mathrm{CH}(\mathrm{OH})-\mathrm{CH}_{2}-\mathrm{OH}$
$\mathrm{HO}-\mathrm{CH}_{2}-\mathrm{CH}_{2}-\mathrm{N}^{+}\left(\mathrm{CH}_{3}\right)_{3}$

codes:

G-OH1 G-1 G-2 G-OH2

$\begin{array}{llll}\mathrm{C}-\mathrm{OH} & \mathrm{C}-1 & \mathrm{C}-2 & \mathrm{C}-3\end{array}$ 
In order to find more about the molecular interactions of the NADES components, we carried out the 2D ROESY and 2D HOESY experiments. The 2D ROESY (Rotating-frame Overhauser SpectroscoPY) experiment is a good method to obtain nuclear Overhauser effect (NOE) information among ${ }^{1} \mathrm{H}$ nuclei in a molecule. The 2D Heteronuclear NOESY (HOESY) experiment allows us to detect heteronuclear through-space NOE connectivities between ${ }^{1} \mathrm{H}$ and ${ }^{13} \mathrm{C}$ nuclei. The ROESY plot (Figure 5c) shows the correlation of water molecules (at $4.4 \mathrm{ppm}$ ) with $\mathrm{C}-\mathrm{OH}, \mathrm{P}-\mathrm{OH} 1$ and $\mathrm{P}-\mathrm{OH} 2$ (at 5.4, 4.85, and $4.85 \mathrm{ppm}$, respectively), probably due to chemical exchange and interactions among them. In addition, $\mathrm{C}-\mathrm{OH}$ and $\mathrm{C}-1$ are correlated due to proximity of those protons; $\mathrm{C}-\mathrm{OH}$ and $\mathrm{C}-3$ seem to be weakly correlated probably due to interactions between them. The HOESY data (Figure 5d) is similar to the HSQC plot (Figure 5b) because not much NOEs was observed between the nuclei. The only correlation that may be of interest is between ${ }^{13} \mathrm{C}$ of $\mathrm{C}-1$ and ${ }^{1} \mathrm{H}$ of $\mathrm{C}-2$, due to the proximity of these nuclei.

\subsubsection{Choline chloride/glycerol/water mixture}

The NMR spectra of this system were previously studied by Hadj-Kali et al [25]. The ${ }^{1} \mathrm{H}$ NMR spectra of 1:3 (molar ratio) choline chloride/glycerol (no added water) and 1:3 choline chloride/glycerol with 25 weight \% water are given in Figures $6 \mathrm{a}$ and $6 \mathrm{~b}$, respectively. Our assignments are similar to those of Hadj-Kali et al [25], except that our water (HOD) signal (in $\mathrm{d}_{6}$-DMSO) occurs at about $3.30-3.42 \mathrm{ppm}$, showing slight downfield shifts with increasing water, whereas larger downfield shifts were reported by Hadj-Kali et al [25]. The 2D NOESY spectrum of the 1:3 choline chloride/glycerol with 25 weight $\%$ water sample was given in Figure 6c. In the 2D plot, correlations among the HOD and the OH peaks in choline chloride and glycerol are observed, probably due to chemical exchange and interactions among them.

Thus, in both the choline chloride/1,2-propanediol/water and the choline chloride/glycerol/water systems, water interacts and exchanges with the hydroxyl groups in NADES components in the range of the water added (50 weight $\%$ or less). Thus, water serves as a diluent for the NADES components, in agreement with earlier studies $[21,25]$.

\subsection{Extraction Studies}

A preliminary study of the extraction of soluble sugars from banana-derived non-starch polysaccharides was carried out with: 1) water/ethanol, and 2) NADES/water mixed system. In both cases, microwaveassisted extraction was used for the extraction of soluble sugars. Water and ethanol gave about $71.5-$ $88.7 \%$ extracted soluble sugars (Table 5). Extractions at $70{ }^{\circ} \mathrm{C}$ showed higher $\%$ extractables than at 25 ${ }^{\circ} \mathrm{C}$; this is not surprising because the solubility of soluble sugars is usually enhanced with heat [26, 27]. In comparison, with NADES/water mixture of malic acid/ $\beta$-alanine/water (molar ratio 1:1:3), microwave-assisted extraction of banana puree at $25^{\circ} \mathrm{C}$ and $30 \mathrm{~min}$ gave the highest quantity of extracted soluble sugars (106.3\%). Thus, the NADES/water system coupled with microwave-assisted extraction represented a much more effective method for this application. A more detailed account of this work will be published in due time.

Table 5. Amount of soluble sugars obtained from banana-derived non-starch polysaccharides through microwaveassisted extraction as a function of extracting solvent, temperature, and time.

\begin{tabular}{clll}
\hline Extracting solvent & Temp $\left({ }^{\circ} \mathrm{C}\right)$ & Time $(\min )$ & \% Extracted $($ dry base $)$ \\
\hline MA/BA/water (molar ratio 1:1:3) & 25 & 30 & 106.3 \\
Ethanol & 25 & 30 & 79.7 \\
Water & 25 & 30 & 71.5 \\
Ethanol & 70 & 30 & 88.7 \\
Water & 70 & 30 & 81.9 \\
\hline
\end{tabular}

\section{Conclusions}

In this work, we examined the solvent properties of several NADES systems and the effect of water addition. The viscosity of NADES decreased exponentially with water addition, whereas density 
decreased linearly. NADES prepared with choline chloride presented the highest conductivities, while NADES with urea showed the lowest values. Choline chloride/sugar and urea/sugar NADES had the highest conductivity at $60 \%$ water dilution. Organic acid/sugar NADES reached the highest value with $80 \%$ water dilution. The refractive index decreased linearly as the water content increased. Thus, water addition can permit some of the NADES solvent properties to be customized, as needed.

Furthermore, we showed that water addition to NADES solvents had a significant effect on the extraction of specific components in food. In particular, NADES with high water content seemed to result in better efficiencies for the extraction of polar compounds in foods. The use of microwave was found to be useful in both the preparation of the NADES solvent system and in the extraction process. Thus, the combination of NADES, water addition, and microwave represents an improved methodology for the extraction of polar compounds from agro-based raw materials, which can decrease solvent viscosity and accelerate extraction.

Three of the 12 principles of green chemistry are: 1) Safer solvents and auxiliaries. 2) Use of renewable feedstocks, and 3) Design for energy efficiency [1, 2]. We believe the use of NADES satisfies the first two principles, and the use of microwave (with the reduction in reaction time) satisfies the third principle. Thus, the approach presented in this work can be considered an example of green chemistry. Variations of this approach should be useful for extraction purposes in food processing.

Acknowledgements. This research was supported by FAPESP (São Paulo Research Foundation) under grant 2016/15783-9. The authors would like to thank the Food Research Center - FoRC for the financial support under FAPESP grant 2013/07914-8.

Mention of trade names or commercial products in this publication is solely for the purpose of providing specific information and does not imply recommendation or endorsement by the U.S. Department of Agriculture. USDA is an equal opportunity provider and employer.

\section{References}

1. P. T. Anastas and J.C. Warner, Green chemistry: Theory and practice. Oxford Univ. Press, 1998.

2. Environmental Protection Agency, Basics of Green Chemistry. Available: https://www.epa.gov/greenchemistry/ basics-green-chemistry

3. P. J. Dunn, "The importance of green chemistry in process research and development," Chemical society reviews, vol. 41, pp. 1452-1461, 2012.

4.S. Rizvi, Separation, Extraction and Concentration Processes in the Food, Beverage and Nutraceutical Industries. Woodhead Publishing Ltd., 2010.

5. F. Chemat, N. Rombaut, A. Meullemiestre, M. Turk, S. Perino, A. Fabiano-Tixier, and M. Abert-Vian, "Review of green food processing techniques. Preservation, transformation, and extraction," Innovative food science $\&$ emerging technologies, vol. 41, pp. 357-377, 2017.

6. H. Li, L. Pordesimo, and J. Weiss, "High intensity ultrasound-assisted extraction of oil from soybeans," Food research international, vol. 37, pp. 731-738, 2004.

7. S.I. Kadioglu, T.T. Phan and D.A. Sabatini, "Surfactant-based oil extraction of corn germ," Journal of American oil chemists' society, vol. 88, pp. 863-869, 2011.

8. D.K. Saxena, S.K. Sharma, S.S. and Sambi, S.S., "Comparative extraction of cottonseed oil by n-hexane and ethanol," ARPN Journal of engineering and applied science, vol. 6, pp. 84-89, 2011.

9. B.G. Terigar, S. Balasubramanian, C.M. Sabliov, M. Lima, and D. Boldor, D. "Soybean and rice bran oil extraction in a continuous microwave system: From laboratory- to pilot-scale," Journal of food engineering, vol. 104, no. 2, pp. 208-217, 2011.

10. N.E. Durling, O.J. Catchpole, J.B. Grey, R.F. Webby, K.A. Mitchell, L.Y. Foo, and N.B. Perry, "Extraction of phenolics and essential oils from dried sage (Salvia officinalis) using ethanol-water mixtures," Food chemistry, vol. 101, pp. 1417-1424, 2007.

11. R. Wang, R. Wang, and B. Yang, "Extraction of essential oils from five cinnamon leaves and identification of their volatile compound composition," Innovative of food science and emerging technologies, vol. 10, pp. 289-292, 2009. 
12. W.A. Wannes, B. Mhamdi, J. Sriti, M.B. Jemia, O. Ouchikh, G. Hamdaoui, M.E. Kchouk, and B. Marzouk, "Antioxidant activities of the essential oils and methanol extracts from myrtle (Myrtus communis var. italica L.) leaf, stem and flower," Food and chemical toxicology, vol. 48, pp. 1362-1370, 2010.

13. Y.C. Chukwumah, L.T. Walker, M. Verghese, M. Bokanga, S. Ogutu, and K. Alphonse, "Comparison of extraction methods for the quantification of selected phytochemicals in Peanuts (Arachis hypogaea)," Journal of agricultural and food chemistry, vol. 55, pp. 285-290, 2007.

14. J. Lako, V.C. Trenerry, M. Wahlqvist, N. Wattanapenpaiboon, S. Sotheeswaran, and R. Premier, "Phytochemicals flavonols, carotenoids and the antioxidant properties of a wide selection of Fijian fruit, vegetables and other readily available foods," Food Chemistry, vol. 101, no. 4, pp. 1727-1741, 2007.

15. P.K. Ramamoorthy and A. Bono, "Antioxidant activity, total phenolic and flavonoid content of Morinda citrifolia fruit extracts from various extraction processes," Journal of engineering science and technology, vol. 2, no. 1, pp. 70-80, 2007.

16. Y. Dai, J. Spronsen, G.-J. Witkamp, R. Verpoorte, and Y.H. Choi, "Natural deep eutectic solvents as new potential media for green technology," Analytica Chimica Acta, vol. 766, pp. 61-68, 2013.

17. Y. Dai, E. Rozema, R. Verpoorte, and Y.H. Choi, "Application of natural deep eutectic solvents to the extraction of anthocyanins from Catharanthus roseus replacing conventional organic solvents," Journal of Chromatography A, vol. 1434, pp. 50-56, 2016.

18. A.V. Gómez, A. Biswas, C.C. Tadini, R.F. Furtado, C.R. Alves, and H. N. Cheng, "Use of natural deep eutectic solvents for polymerization and polymer reactions," Journal of Brazilian chemical society, submitted.

19. A. Farran, C. Cai, M. Sandoval, Y. Xu, J. Liu, M.J. Hernaiz, and R.J. Linhardt, "Green solvents in carbohydrate chemistry: From raw materials to fine chemicals," Chemical reviews, vol. 115, pp. 6811-6853, 2015.

20. M.C. Gutierrez, M.L. Ferrer, C.R. Mateo, and F. del Monte, "Freeze-drying of aqueous solutions of deep eutectic solvents: A suitable approach to deep eutectic suspensions of self-assembled structures," Langmuir, vol. 25, pp. 5509-5515, 2009.

21. Y. Dai, G.-J. Witkamp, R. Verpoorte, and Y.H. Choi, "Tailoring properties of natural deep entectic solvents with water to facilitate their applications," Food chemistry, vol. 187, pp. 14-19, 2015.

22. T. Masuko, A. Minami, .N. Iwasaki, T. Majima, S.,Nishimura, and Y.C. Lee, "Carbohydrate analysis by a phenol-sulfuric acid method in microplate format," Analytical biochemistry, vol. 339. pp. 69-72, 2005.

23. H. N. Cheng, "NMR characterization of polymers," in Modern Methods of Polymer Analysis. Wiley, 1991, pp. 409-493.

24. NMRShiftDB, Available: http://nmrshiftdb.nmr.uni-koeln.de/

25. M.K. Hadj-Kali, K.E. Al-khidir, I. Wazeer, L. El-blidi, S. Mulyono, and I.M. AlNashef, "Application of deep eutectic solvents and their individual constituents as surfactants for enhanced oil recovery," Colloids and Surfaces A: Physicochemical and Engineering Aspects, vol. 487, pp. 221-231, 2015.

26. M.C. Gray, A.O. Converse, and C.E. Wyman, "Sugar monomer and oligomer solubility data and predictions for application to biomass hydrolysis," Applied Biochemistry \&3 Biotechnology, vol. 105-108, pp. 179-193, 2003.

27. F. Jia, J. Chawhuaymak, M.R. Riley, W. Zimmt, and K.L. Ogden, "Efficient extraction method to collect sugar from sweet sorghum," Journal of biological engineering, vol. 7, paper 1, 2013.

Available: https://doi.org/10.1186/1754-1611-7-1. 